\title{
New Insight in the Assessment of Left Ventricular Function in Paradoxical Low Flow Aortic Stenosis Patients with Normal Left Ventricular Ejection Fraction: A Mini-Review
}

\begin{abstract}
Sherif Baath Allah', Enrico Mangieri'2, Jan Fedacko3, Petras Lohana ${ }^{4}$, Mohammed Elmahal,6, Amena Elsaady ${ }^{3}$, Madian Abdelrahman7, Jaipaul Singh ${ }^{8}$, Mohamed Khorshid ${ }^{9}$, Ram B. Singh ${ }^{10}$, Galaleldin Nagib Elkilany11,12*
\end{abstract}

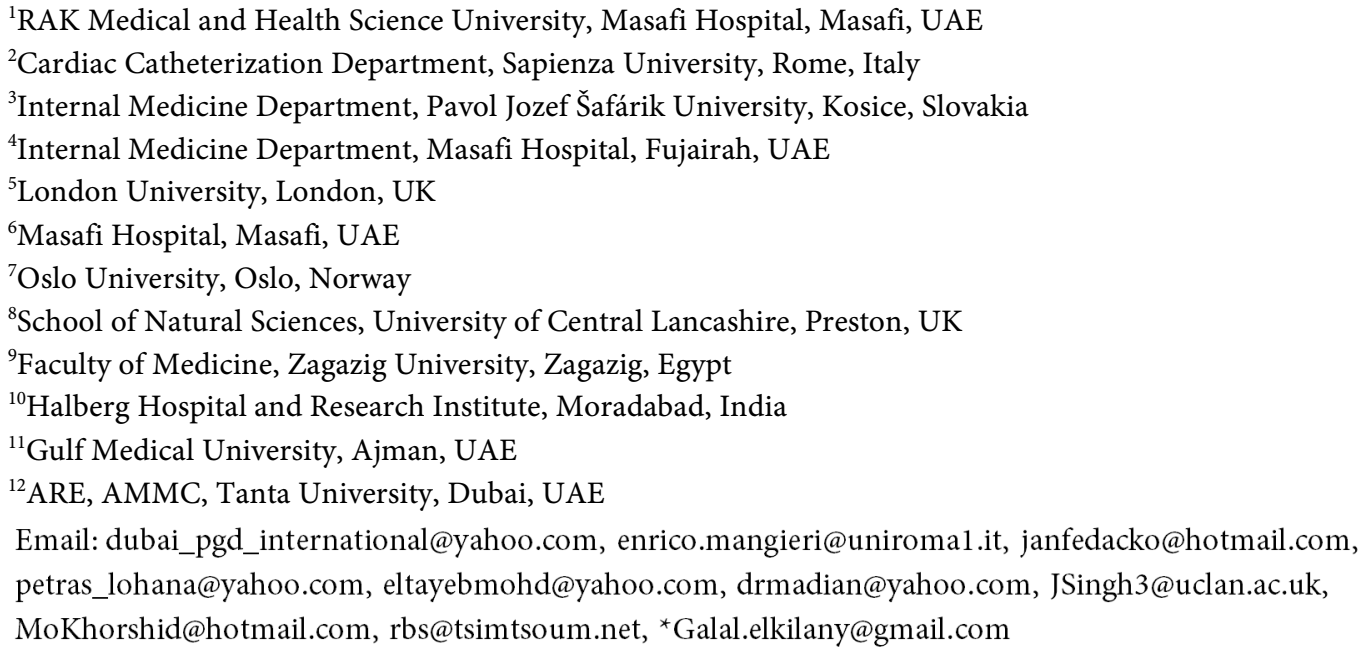

How to cite this paper: Allah, S.B., Mangieri, E., Fedacko, J., Lohana, P., Elmahal, M., Elsaady, A., Abdelrahman, M., Singh, J., Khorshid, M., Singh, R.B. and Elkilany, G.N. (2020) New Insight in the Assessment of Left Ventricular Function in Paradoxical Low Flow Aortic Stenosis Patients with Normal Left Ventricular Ejection Fraction: A Mini-Review. World Journal of Cardiovascular Surgery, 10, 264-270. https://doi.org/10.4236/wjcs.2020.1012028

Received: November 9, 2020 Accepted: December 18, 2020 Published: December 21, 2020

\begin{abstract}
Paradoxical valvular aortic stenosis (VAS) is a challenging area of clinical cardiology for the practitioners. It involves a small aortic valve area, low flow rate and mean pressure gradient although there is normal left ventricular ejection fraction. The aim of this study was to assess left ventricular (LV) dysfunction in a symptomatic severe aortic valve stenosis which is of crucial importance in identifying patients at risk of heart failure, postoperative complications and increased mortality. There are new insights which are involved in assessment of LV myocardial function including global longitudinal strain (GLS) by two-dimensional speckle tracking echocardiography (2D STE), myocardial performance index (MPI) and maximum rate of LV pressure rise $(+\mathrm{dP} / \mathrm{dt})$ during isovolumetric contraction time of the LV. This information can provide both diagnostic and prognostic information in addition to stan-
\end{abstract}


Copyright $\odot 2020$ by author(s) and Scientific Research Publishing Inc. This work is licensed under the Creative Commons Attribution International License (CC BY 4.0).

http://creativecommons.org/licenses/by/4.0/

Open Access dard echocardiographic and clinical parameters. However, a profound understanding of the complex interaction between loading conditions, chamber geometry and contractility is necessary for the correct interpretation of myocardial deformation in order to draw appropriate conclusions in patients with aortic valve disease. This mini review is related to new and novel insights into the assessment of left ventricular function (LVF) in paradoxical low flow aortic stenosis patients with normal left ventricular ejection fraction (LVEF).

\section{Keywords}

Aortic Stenosis, LVEF, Aortic Valve, Myocardial Function, Global Longitudinal Stain, Echocardiography

\section{Introduction}

The most challenging finding in clinical practice is associated with paradoxical severe valvular aortic stenosis (VAS). It is an aortic valve area (AVA) of $<1 \mathrm{~cm}^{2}$ with a peak velocity of $<4 \mathrm{~m} / \mathrm{s}$ and a mean pressure gradient of $<40 \mathrm{~mm} \mathrm{Hg}$ despite normal LVEF. Subclinical myocardial dysfunction that is characterized by impaired left ventricular (LV) global longitudinal systolic strain (GLS) is often present in patients with a symptomatic severe AS with preserved LVEF. This is due to LVEF which is highly dependent on preload and afterload and its depression seems to occur at a very late stage in many valvular-induced heart diseases.

\section{Application of Deformation Imaging and Myocardial Performance in Clinical Practice}

The entity of "paradoxical" low flow and low gradient AS with preserved LVEF refers to patients with hypertrophied, small ventricles resulting in reduced trans-valvular flow for which stroke volume index (SVi) of $<35 \mathrm{~mL} / \mathrm{m}^{2}$ is a surrogate despite normal LVEF [1]. However, this entity has to be diagnosed with particular care as there may be other small valve area and low gradient in the presence of normal LVEF. This may be more likely to occur during technical factors in AVA calculation ((error in measurements of left ventricular outflow (LVOT) diameter)) and as such they have to be carefully excluded. So, the main pitfalls associated with conventional transthoracic echocardiographic diagnosis of paradoxical low flow low gradient AV stenosis are an error in calculation of the SV due to inaccurate measurements of LVOT diameter (TTE tends to underestimate the diameter of LVOT partly due to elliptical rather than circular anatomy and in the presence of extensive calcification) and/or misplacement (misalignment) of pulsed-wave Doppler sample volume (too low in the LVOT or too lateral towards the anterior mitral valve leaflet may lead to underestimation of flow velocity and thus low SV, whereas a position too close to the valve or to the septum may lead to overestimation) [1]. This mini review has relevant clinical application: in the assessment of left ventricular function. Severe "paradoxi- 
cal" low flow low gradient AS with preserved LVEF has in commonly more prevalent in patients of older age, in women, and in patients with concomitant system arterial hypertension (HTN) [1]. Reduced longitudinal LV function and fibrosis have been found in many cases (see Figure 1). However, the vast majority of these patients had a history of HTN that may also have caused the LV remodeling (hypertrophy and fibrosis) [1] [2]. Furthermore, uncontrolled arterial hypertension can affect the flow and pressure gradient values in severe aortic valve stenosis. This entity is most frequently characterized by restrictive physiology in relation to more pronounced LV concentric remodeling, reduced, LV cavity size, impaired LV filling, and reduced systemic arterial compliance [3]. Accordingly, diastolic dysfunction and filling pressure (left atrial/left ventricular end diastolic pressure. (LVEDP)) should be initially assessed non-invasively by conventional mitral Doppler flow, tissue Doppler imaging (TDI), left atrial pressure $(\mathrm{E} / \mathrm{Ea})$, and pulmonary venous flow as illustrated in Figure 1.

Alternative to LVEF, the following techniques are able to assess LV myocardial function accurately in low flow AS patients with normal EF and they include:

1) Mitral annular displacement by TDI $<12 \mathrm{~mm}$;

2) Global longitudinal strain of the LV (GLS) by 2D STE (see Figure 1 \& Figure 2) [4];

3) Myocardial Performance index (Tei Index) > 0.42. 58 - 63 (see Figure 3; [5]-[10]);

4) BNP levels $>550 \mathrm{pg} / \mathrm{ml}[11]$ and;

5) Maximum rate of LV pressure development (see Figure 4) [12].

\section{Left Ventricular Global Longitudinal Strain (LV GLS)}

A recent meta-analysis study [13] demonstrates that in asymptomatic patients with significant AS and normal LVEF, impaired (LV) GLS is associated with reduced survival time. These data emphasize the potential usefulness of LV GLS for risk stratification and management of these patients (see Figure 2) [13]. This meta-analysis included 10 studies, 1067 asymptomatic patients with significant AS and LVEF > 50\% were analyzed. The median of left ventricular (LV) GLS was

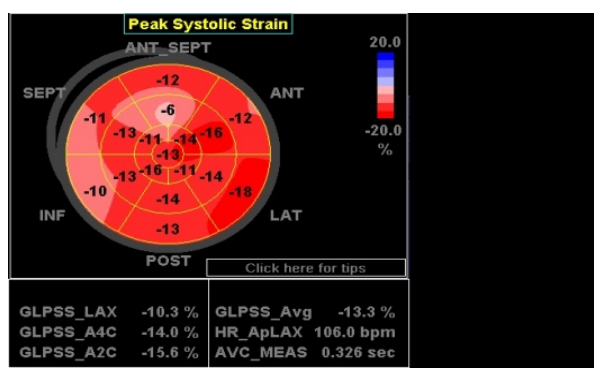

(a)

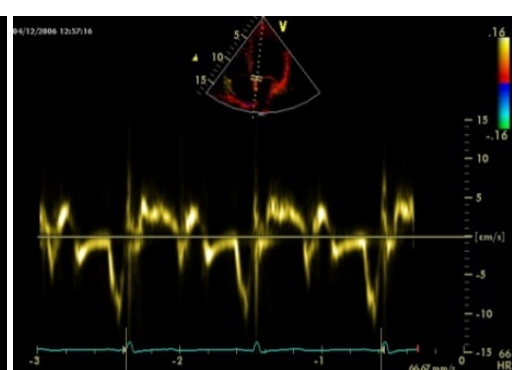

(b)

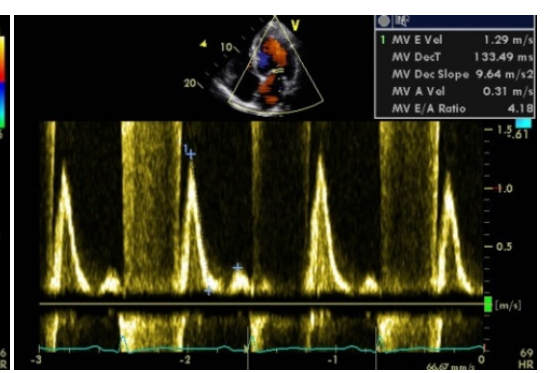

(c)

Figure 1. This figure illustrates the role of global longitudinal systolic strain (GLS) in the diagnosis of subclinical systolic dysfunction-and normal LVEF in arterial hypertension. (a) shows 2D Strain for detection of subclinical LV dysfunction and normal EF (GLPSS-13\%), in hypertensive patient with low GLS in AS patient with paradoxical low flow-low gradient severe AS. (b) shows grade III/IV diastolic dysfunction by conventional Doppler flow and (c) shows evidence of diastolic dysfunction by tissue Doppler imaging $(\mathrm{Ea}<5 \mathrm{~cm} / \mathrm{s})$. 

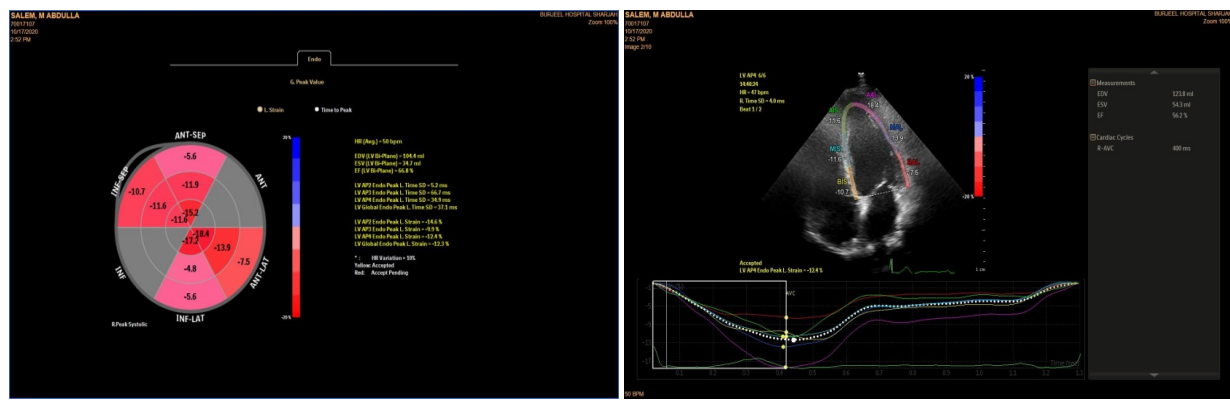

Figure 2. Global LONGITUDINAL Strain by $2 \mathrm{D}$ speckle tracking echocardiography showing depressed GLS $(-12.4 \%)$ in the presence of normal LVEF $(56.2 \%)$ in paradoxical low flow AS (taken from reference [14]).

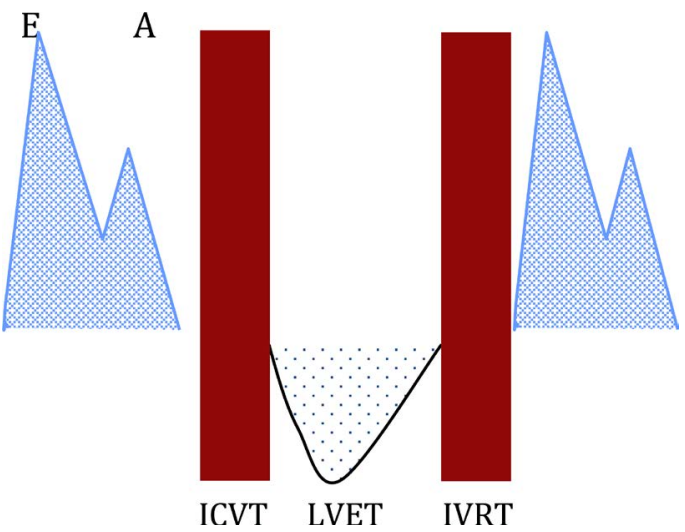

Figure 3. Schematic diagram showing how to measure index of myocardial performance (MPI): isovolumetric relaxation time (IVRT), isovolumetric contraction time (IVCT), LV ejection time (LVET), MPI = IVRT + IVCT/LVET.

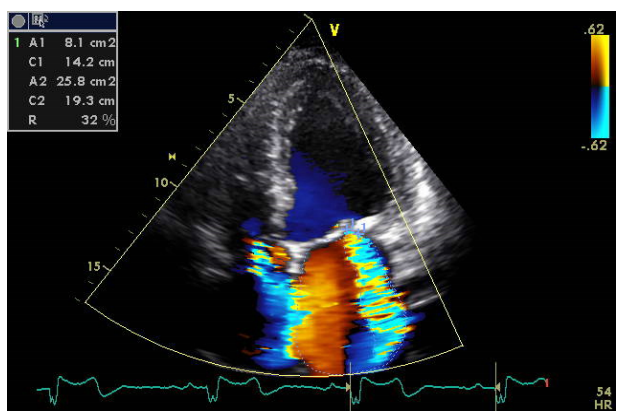

(a)

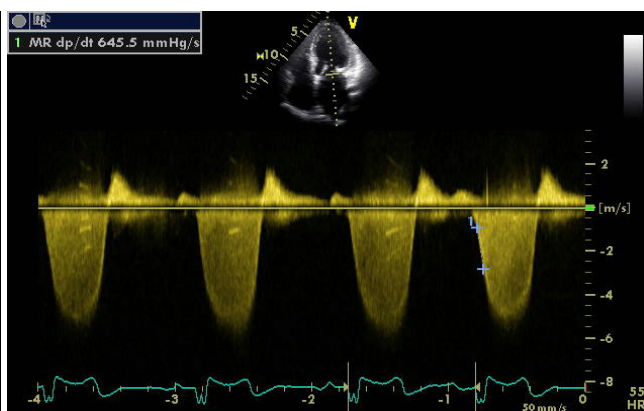

(b)

Figure 4. (a) 4 chambers $2 \mathrm{D}$ echocardiography showing moderate mitral valve incompetence in a patient with AS but normal ejection fraction ( $\mathrm{EF}=51 \%)$. (b) shows that maximum rate of $\mathrm{LV}$ positive pressure development $(+\mathrm{dP} / \mathrm{dt})$ which is depressed in the same patient $(645 \mathrm{~mm} \mathrm{Hg})$ and with measurement of $\mathrm{dP} / \mathrm{dt}$ at $1^{\text {st }}$ and $3^{\text {rd }} \mathrm{m} /$ seconds of mitral regurgitation (MR) continuous wave Doppler (CWD) slope (taken from reference [12]).

$-16.2 \%$ (from $-5.6 \%$ to $-30.1 \%$ ). There were 91 deaths reported during follow-up with median of 1.8 (0.9 to 2.8 ) years, resulting in a pooled crude mortality rate of $8.5 \%$. The LV GLS performed well in the prediction of death (area under the curve: 0.68$)$. The best cut off value identified was LV GLS of $-14.7 \%$ (sensitivity, 60\%; specificity, 70\%). Using random effects model, the risk of death 
for patients with LV GLS $<-14.7 \%$ ( $\mathrm{p}<0.0001$ ), without significant heterogeneity between studies ( $\mathrm{I} 2=18.3 \% ; \mathrm{p}=0.275)$. The relationship between LV GLS and mortality remained significant in patients with LVEF $\geq 60 \%(\mathrm{p}=0.001)$. Similarly, Lancellotti et al. [11] examined a cohort of 163 patients with at least moderate to severe, asymptomatic AS. They demonstrated that impaired LV longitudinal myocardial deformation was an independent predictor of survival. Those patients with longitudinal strain $>-15.9 \%$ had significantly better outcome than patients with the strain below $-15.9 \%$ (4-year survival of 63 vs. $22, \mathrm{p}$ $<0.001)$.

Interestingly, Elkilany et al. [14] found a concomitant dilated cardiomyopathy which was observed in $1.8 \%$ of subjects with bicuspid aortic valve (BAV). A few different clinical and echocardiographic characteristics were found. They concluded that, the presence of cardiomyopathy was independently associated with heart failure either clinically or at the sub clinical stage, which can be identified by global systolic strain.

\section{Myocardial Performance Index (MPI)}

The Doppler-derived myocardial performance index (MPI) has been considered as a diagnostic and prognostic Doppler marker for many different clinical conditions. TDI-MPI and PWD-MPI were significantly higher in patients with grade I diastolic dysfunction (DDI) than in control subjects: $0.49 \pm 0.14$ vs. 0.40 \pm 0.09 ( $\mathrm{p}<0.001)$ and $0.45 \pm 0.11$ vs. $0.37 \pm 0.08$ ( $\mathrm{p}<0.001)$, respectively. Cutoff values of TDI-MPI $>0.42$ and PWD-MPI $>0.40$ identified DDI subjects, with sensitivities of $74 \%$ and $64 \%$; specificities of $61 \%$ and $69 \%$ [5].

Initial diastolic dysfunction detected by Doppler echocardiography is an independent risk factor for the development of heart failure and all-cause mortality, even in asymptomatic patients [6]. The myocardial performance index (MPI) or Tei-Index, described more than a decade ago, has been well documented in the literature as a prognostic and progression marker for various heart diseases [6] [7] [8]. However, in the majority of these studies, MPI was used in patients with combined systolic and diastolic dysfunctions. One limitation of the conventional Doppler-derived Myocardial Performance Index (PWD-MPI) method is that the measures of time intervals which are based on flow-velocity curves and are performed in different cardiac cycles. This method requires several measurements to reduce beat-to-beat variation. An alternative for MPI calculation is the use of the pulsed-wave tissue Doppler imaging-derived myocardial performance index (TDI-MPI), which allows simultaneous measurement of both the diastolic and systolic intervals in the same cardiac cycle, with high diagnostic accuracy in subjects with heart failure and left-ventricular dysfunction [9] [10].

\section{Dobutamine Stress Echocardiography (DSE)}

Low dose DSE may be used to rule-out pseudo-severe AS, but may not be applicable or conclusive in a significant proportion of patients with paradoxical low 


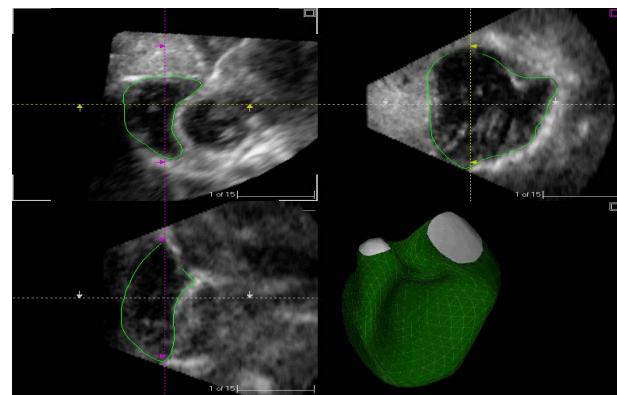

(a)

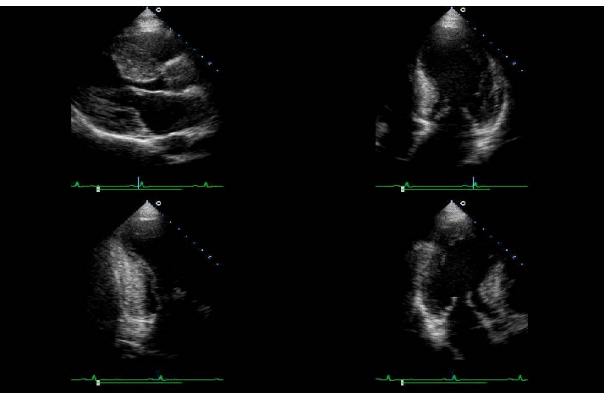

(b)

Figure 5. 3D echocardiography (a) during DSE (b) is feasible in the majority of pts with severe low flow low gradient AS to identify pseudo low flow but severe critical AS, 3DE is able to estimate accurately the LVEF and SV index at rest and during peak stress.

flow low gradient AS, particularly in those patients with Doppler evidence of restrictive LV physiology (restrictive filling pattern) (Figure 5) [15] [16].

In conclusion, the implication of cardiac ultrasound with routine use of GLS via $2 \mathrm{D}$ STE is a gold standard for the early detection and proper management of cardiac dysfunction in a symptomatic severe AS patients and this is reasonably recommended in BAV patients. In addition, an accurate assessment of LV contractility is feasible by MPI and maximum rate of LV pressure development. The depression of these parameter values is considered an independent risk factor for the development of heart failure and all-cause mortality, even in asymptomatic patients and the need for early intervention (aortic valve replacement).

\section{Conflicts of Interest}

The authors declare no conflicts of interest regarding the publication of this paper.

\section{References}

[1] Pibarot, P. and Dumesnil, J.G. (2012) Low-flow, Low-Gradient Aortic Stenosis with Normal and Depressed Left Ventricular Ejection Fraction. Journal of the American College of Cardiology, 60, 1845-1853. https://doi.org/10.1016/j.jacc.2012.06.051

[2] Baumgartner, H., Hung, J., Bermejo, J., Chambers, J.B., Edvardsen, T., et al. (2017) Recommendations on the Echocardiographic Assessment of Aortic Valve Stenosis: A Focused Update from the European Association of Cardiovascular Imaging and the American Society of Echocardiography. European Heart Journal-Cardiovascular Imaging, 18, 254-275. https://doi.org/10.1093/ehjci/jew335

[3] Pibarot, P. and Dumesnil, J.G. (2013) Paradoxical Low-Flow, Low-Gradient Aortic Stenosis: New Evidence, More Questions. Circulation, 128, 1729-1732. https://doi.org/10.1161/CIRCULATIONAHA.113.005718

[4] Elkilany, G.E.N., Allah, S.B., Fedacko, J., Singh, J., Nanda, N.C. and Salama, M. (2019) Hypertensive Cardiomyopathy: Diagnostic Approach and Clinical Differentiation from Hypertrophic Cardiomyopathy. Journal of Cardiology and Cardiovascular Therapy, 15, Article ID: 555919.

[5] Maria, J., de Oliveira, B., Romero, I., et al. (2019) Clinical Value of Myocardial Performance Index in Patients with Isolated Diastolic Dysfunction. Cardiovascular UI- 
trasound, 17, Article No. 17. https://doi.org/10.1186/s12947-019-0167-x

[6] Redfield, M.M., Jacobsen, S.J., Burnett Jr., J.C., et al. (2003) Burden of Systolic and Diastolic Ventricular Dysfunction in the Community: Appreciating the Scope of the Heart Failure Epidemic. JAMA, 289, 194-202.

https://doi.org/10.1001/jama.289.2.194

[7] Niemann, M., Breunig, F., Beer, M., et al. (2011) Tei Index in Fabry Disease. Journal of the American Society of Echocardiography, 24, 1026-1032. https://doi.org/10.1016/j.echo.2011.05.021

[8] Liu, D., Hu, K., Herrmann, S., Cikes, M., et al. (2017) Value of Tissue Doppler-Derived Tei Index and Two-Dimensional Speckle Tracking Imaging Derived Longitudinal Strain on Predicting Outcome of Patients with Light-Chain Cardiac Amyloidosis. The International Journal of Cardiovascular Imaging, 33, 837-845. https://doi.org/10.1007/s10554-017-1075-5

[9] Meric, M., Yesildag, O., Yuksel, S., et al. (2014) Tissue Doppler Myocardial Performance Index in Patients with Heart Failure and Its Relationship with Haemodynamic Parameters. The International Journal of Cardiovascular Imaging, 30, 1057-1064. https://doi.org/10.1007/s10554-014-0449-1

[10] Duzenli, M.A., Ozdemir, K. and Aygul, N. (2009) Comparison of Myocardial Performance Index Obtained Either by Conventional Echocardiography or Tissue Doppler Echocardiography in Healthy Subjects and Patients with Heart Failure. Heart Vessel, 24, 8-15. https://doi.org/10.1007/s00380-008-1069-2

[11] Lancellotti, P., Moonen, M., Magne, J., O’Connor, K., Cosyns, B., Attena, E., et al. (2010) Prognostic Effect of Long-Axis Left Ventricular Dysfunction and B-Type Natriuretic Peptide Levels in Asymptomatic Aortic Stenosis. The American Journal of Cardiology, 105, 383-388. https://doi.org/10.1016/j.amjcard.2009.09.043

[12] Elkilany, G.E.N., De Groef, M. and Kabbash, I. (2011) How to Identify Latent Systolic Dysfunction and Post-Operative Risk in Patients with Mitral Incompetence and Normal Ejection. World Journal of Cardiovascular Surgery, 1, 11-17. https://doi.org/10.4236/wjcs.2011.12003

[13] Magne, J., Cosyns, B., Bogdan, A., et al. (2019) Distribution and Prognostic Significance of Left Ventricular Global Longitudinal Strain in Asymptomatic Significant Aortic Stenosis: An Individual Participant Data Meta-Analysis. JACC: Cardiovascular Imaging, 12, 84-92. https://doi.org/10.1016/j.jcmg.2018.11.005

[14] Elkilany, G.E.N., Ghobashi, A., Salama, M., Singh, J., et al. (2020) Prevalence and Sub Clinical Detection of Concomitant Dilated Cardiomyopathy in Subjects with Bicuspid Aortic Valves. Journal of Cardiology and Cardiovascular Therapy, 16, 4-8.

[15] Clavel, M.A., Ennezat, P.V., Maréchaux, S., et al. (2013) Stress Echocardiography to Assess Stenosis Severity and Predict Outcome in Patients with Paradoxical Low-Flow, Low-Gradient Aortic Stenosis and Preserved LVEF. JACC: Cardiovascular Imaging, 6, 175-183. https://doi.org/10.1016/j.jcmg.2012.10.015

[16] Pibarot, P. and Clavel, M.-A. (2015) Management of Paradoxical Low-Flow, Low-Gradient Aortic Stenosis. Journal of the American College of Cardiology, 65, 67-71. https://doi.org/10.1016/j.jacc.2014.10.030 